DOI:10.5392/I.JoC.2010.6.3.071

\title{
Natural Sections in Product Design
}

\author{
Tom Page \\ Electronic Product Design in Department Design and Technology \\ Loughborough University, Loughborough, Leicestershire, United Kingdom
}

Gisli Thorsteinsson

Design and Craft Education Department

University of Iceland, Stakkahlid, Reykjavik, Iceland

Joong Gyu Ha

Industrial Design in Fine Art Education Department

Gyeongsang National University, Jinju, Gyeongnam, Korea

\begin{abstract}
The golden ratio is a mysterious number that surprisingly appears in science, physics, mathematics, as well as in nature. The number 1.618 seems to be a universal constant, and crops up whenever the subject is of beauty or elegance. Beautiful flowers and sea shells and also attractive people have a common number and that is 1.618 or $\varphi$ (phi). This paper does a study into the story of phi, and describes how the golden ratio is derived. Artists, architects and designers have employed the ratio into dimensioning their works of art to achieve visual appeal. Examples such as the Greek Parthenon of the Acropolis and paintings such as the Last Supper all use this magic number. An investigation was conducted among 50 people to test if looking at golden proportioning was actually appealing, or if it was just a type among overzealous enthusiasts. The results show that the golden ratio may actually be of some use
\end{abstract}

Keywords: Golden Ratio, Golden Proportion, Golden Ratio in Nature and Arts, Golden Ratio in Industrial Design, Golden Proportion in Product Design, Investigation of the Golden Ratio in Products

\section{INTRODUCTION}

\subsection{General Appearance}

The golden ratio, golden constant, golden section, golden mean, golden proportion, extreme to mean ratio, the divine proportion and the divine section are all names for the mysterious ratio, a mathematical constant that keeps occurring in mathematics and nature. It has therefore been regarded as a useful ratio to be used in design, where designers believe this proportioning tool can produce aesthetically pleasing designs. The golden ratio is an irrational constant approximately 1.618 and given the symbol $\varphi$ (phi). The definition of the golden ratio will be given in Section 2 along with methods of calculation and the derivation of important parts such as the golden section and the golden rectangle. The different terms for phi listed above will be used interchangeably throughout this paper.

In Section 3 there will be an extensive review on the literature and prior art on the topic. The views from the different authors will be discussed. Examples of cases and

\footnotetext{
*Corresponding author. E-mail : hajoonggyu@gnu.ac.kr Manuscript received Jun. 04, 2010 ; accepted Jul. 14, 2010
}

discoveries of the golden ratio in existing applications will be in Section 4. This section is split into two sections, one for the discoveries of the golden ratio within nature and one for the application within the arts.

The final sections of this paper will be on the investigation that will be conducted to test if the golden ratio actually affects product appeal. The methodology in the creation of the questionnaire will be addressed, and the results will be tallied evaluated.

At the end of this paper it should be clear if the golden ratio should be a factor within industrial design.

\section{BACKGROUND}

\subsection{Specifications}

In this section the golden ratio will be explained and the method to calculate it will be shown.

The golden section is a line segment sectioned into two according to the golden ratio, (see Fig.1.). The line is sectioned in a way that the ratio of $a+b: a$ is the same as a:b. This ratio is equal to 1.618 to 3 decimal places and it is called the golden 
constant. The golden constant is an irrational number and has an infinite amount of decimal places.[1]

The golden ratio is when a line in ratio to another line can be illustrated as 1:1.618.

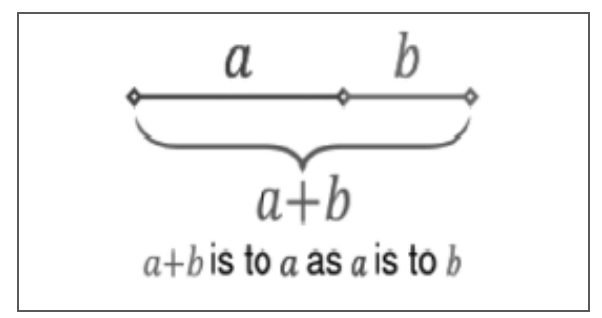

Fig.1. Golden Section

The golden section can be expressed algebraically (see Fig.2.)

$$
\varphi=\frac{1+\sqrt{5}}{2} \approx 1.6180339887 \ldots
$$

Fig.2. Golden Section in Algebra

The golden ratio is particularly useful in geometry, where designers have used it as a proportioning tool. The most simplest of shapes that can be created from the golden ratio is the golden rectangle. Fig.3. is a diagram that shows how a golden rectangle can be created. The following steps to create it in this way are:

1) Construct a unit square.

2) Draw a line from the midpoint of one side to an opposite corner.

3) Use that line as the radius to draw an arc that defines the long dimension of the rectangle.

Other shapes that can use the golden ratio include the golden triangle, ellipse, and spirals. A golden triangle is one where it is an isosceles triangle with base angles of $72^{\circ}$ and a vertex of $36^{\circ}$. A golden ellipse is created by inscribing an ellipse within a golden rectangle with the ratio of its minor and major axis as $1: 1.618$.

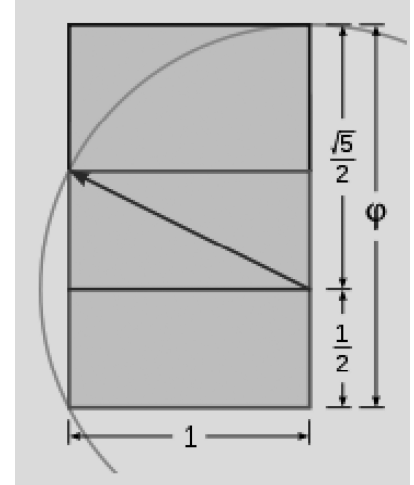

Fig.3. Construction of Golden Rectangle

The regular pentagon is a shape that has special properties that relate to the golden ratio. A regular pentagon is a five sided shape that can be inscribed within a circle.
The pentagon can be split up into three triangles with the acute golden triangle in the middle, and the obtuse golden triangles either side. The pentagon is related to the golden ratio in which when a line is drawn connecting two corners, the ratio of $\mathrm{AB}: \mathrm{BG}$ is equal to the golden constant, (see Fig.4.).

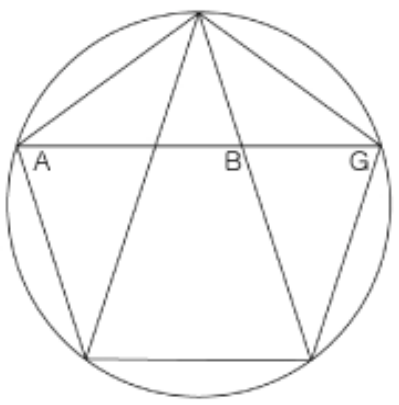

Fig.4. Golden Pentagon

The golden constant is such a mysterious number because it has appeared in many mathematical calculations and sums, and even in studies in nature and science. One interesting discovery is that the golden constant appears when looking at the ratios of Fibonacci numbers. The Fibonacci sequence is a sequence of numbers that uses the addition of the previous two numbers to make the next one. The Fibonacci sequence goes as follows: $1,1,2,3,5,8,13,21,34,55, \ldots$ etc. It can be seen that the third number in the sequence is indeed the sum of the first two $1+1$ $=2$, and the next number is calculated by doing $1+2=3$, and the next is $2+3=5$ and so on. Below is a table that shows how the ratio of successive Fibonacci numbers converges to phi (for the first 20 Fibonacci numbers). This is why the Fibonacci sequence is always mentioned with the golden ratio.

$\begin{array}{llll}1 & : & 1 & =1.00000 \\ 2 & : & 1 & =2.00000 \\ 3 & : & 2 & =1.50000 \\ 5 & : & 3 & =1.66666 \\ 8 & : & 5 & =1.60000 \\ 13 & : & 8 & =1.62500 \\ 21 & : & 13 & =1.61538 \\ 34 & : & 21 & =1.61904 \\ 55 & : & 34 & =1.61764 \\ 89 & : & 55 & =1.61818 \\ 144 & : & 89 & =1.61797 \\ 233 & : & 144 & =1.61805 \\ 377 & : & 233 & =1.61802 \\ 610 & : & 377 & =1.61803 \\ 987 & : & 610 & =1.61803\end{array}$

\section{LITERATURE REVIEW}

There is much literature on the subject of the golden constant, dating back as far as $265 \mathrm{BC}$ where Euclid gave the first recorded definition in his book "Elements". The key literature thereafter includes "De Divina Proportione" by Pacioli, 1509, where he describes it as the 'Divine Proportion'; and "Goldener Schnitt" by Ohm, 1835, describing as the 'Golden Section'. It was not only until the twentieth century in 1909 when Mark Barr, an American mathematician, gave the golden ratio the 
symbol $\varphi$ (phi) dedicated after the Greek sculptor Phidias who used it extensively in his work.

The golden ratio came about naturally as it was a number that occurred frequently in mathematics and nature. Much like the well known number $\pi$ (pi), $\varphi$ is also an irrational constant that has been linked to having mysterious properties. It is a number that marries science to aesthetics, where if carefully used the designer can produce a masterpiece created upon mathematical reasoning. The golden ratio has been described as "the universal law in which is contained the ground-principle of all formative striving for beauty and completeness in the realms of both nature and art" [12].

Every piece of literature that touches on the golden ratio will mention its ancient Greek origins, as that was the period where the mathematician Euclid gave its definition, and also the period where famous buildings like the Parthenon were claimed to be designed with. However, Keith Devlin, 2005, said that, "Certainly, the oft repeated assertion that the Parthenon in Athens is based on the golden ratio is not supported by actual measurements. In fact, the entire story about the Greeks and golden ratio seems to be without foundation." [2]. It is known that Euclid explained how to calculate the golden ratio, but there isn't evidence that magnificent structures like the Parthenon used it. Furthermore, many websites and books that illustrate how buildings and products that use the golden ratio of proportioning seem to be only the author's interpretation. However, there are artists and designers that have consciously used the golden ratio which have been documented.

The argument of the effectiveness of applying the golden ratio to design is subjective. It was shown that the golden ratio is a number that occurs in nature, such as the logarithmic spirals of a seashell or the spiralling seeds of a sunflower head. It has also been seen in the spirals of DNA structure and the intervals of the human heartbeat. Thus the connotation of something so natural and elegant that can be governed by mathematics was discovered. Designers, painters and architects used this phenomenon in their works and can be consciously satisfied that its aesthetics would be appealing to everyone. But one of the key questions of the paper that would be addressed is "Does the application of the golden ratio actually affect product appeal?". Fechner, a German psychologist, conducted an experiment in 1876 on people to choose the most pleasing rectangle out of a variety of 10 sizes. It was found that the results had a peak ratio of width and length at 1.62, the golden ratio. Fechner then went onto measuring everything rectangular and found that the ratios average to yet again the golden ratio. Have the like of designers used the golden ratio subconsciously because it is the universal law to aesthetics? [12]. Or is it a misconception built up by followers of the magical number that its frequent occurrence is more of a coincidence? As Livio says in his "Golden Ratio and Aesthetics online article in 2002, "If you have the patience to juggle and manipulate the numbers in various ways, you are bound to come up with some ratios that are equal to the Golden Ratio." [9].

The most recent and well written book about the history of the golden ratio is Mario Livio's "The Golden Ratio" published in 2002. Livio gives an in depth review of the history of mathematics, geometry and then the story of the golden ratio [10]. The difference from his book compared to others is that he does not hesitate to debunk myths that have been created by people who are obsessed with the golden ratio. He calls these people "Golden Numberists", which desperately find and juggle numbers until they are blinded with evidence of a golden ratio match-up. "Golden Numberists" can be found all over the internet, especially on websites set up for golden ratio enthusiasts. On these websites there are many examples of discoveries of the golden ratio; however, most of them are speculative to say the least. For example in Fig.5. it can be seen that a golden section can be seen in the design of the car, but the car is not even facing in particular elevation view i.e. facing front or to the right, so the measurement should not be valid. Furthermore, it is a measurement of a picture which can easily be skewed and cropped to fit within the golden sections. Even though the purpose of the golden ratio is to enhance the visual attractiveness, and for this car it is at this particular angle, it is not as intriguing as those found in nature.

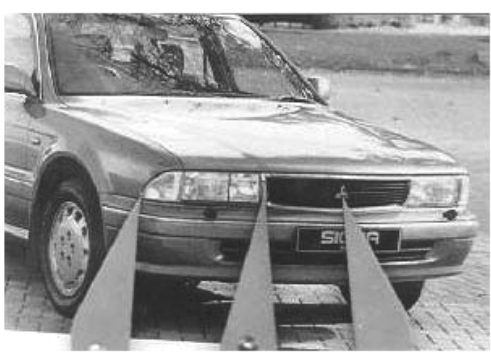

Fig.5. Golden Section on car

Livio has researched and summarised his findings and with each myth the answer is usually that there is no evidence that the designer had known of such phenomenon. But he finds it intriguing how such a constant keeps appearing within nature and summarises that the delight that we derive from this concept is due to the element of surprise. To conclude his last section "Is God a Mathematician?" he writes, "The Golden Ratio is a product of humanly invented geometry. Humans had no idea, however, into what magical fairyland this product was going to lead them. If geometry had not been invented at all, then we might have never known about the Golden Ratio. But then, who knows? It might have emerged as the output of a short computer program." (Livio, 2002:253) [10].

Livio suggests that the golden ratio is not so special. Humans are easily surprised at things that seem to arise in patterns, and will believe in anything mysterious as we live in an age where psychic phenomena sells. But the real truth about the golden ratio is not yet known, more research is yet to be done.

\section{THE GOLDEN RATIO IN EXISTING APPLICATIONS}

The golden ratio has been used and discovered in many existing applications, and these can be categorised into two groups: in nature and in the arts. In this section there will be summaries on creations that have been interpreted with the 
golden ratio.

\subsection{In Nature}

The following sections addresses on creations of nature that appear to follow the golden ratio. Some of these cases are subjective as they do not have credible sources. However, anything that claims it follows the golden ratio is usually just interpretations.

4.1.1 The Human Body The human body is an interesting subject because it seems to have many cases on the use of the golden ratio.

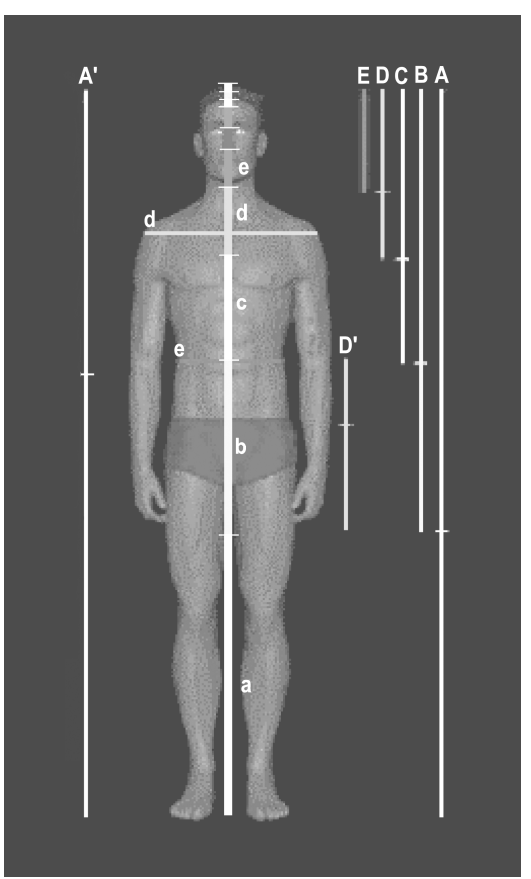

Fig.6. Golden Section in Human Body

Fig.6. shows an image of the human body with the golden proportions between different parts of the body.

- The A line and A' line are the height of the body.

- The B line is the distance from the top of the head to the finger tips and the $\mathrm{b}$ line is also the distance from the naval to the base of the feet, which are golden sections of the A line.

- The $\mathrm{C}$ line is the distance from the top of the head to the naval and elbows, which is a golden section of the B line.

- The D line is the distance from the top of the head to the shoulders, the $d$ line is also the shoulder width and the D' line is also the distance from the elbows to the finger tips. These are all in golden ratio of the $\mathrm{C}$ line.

- The $\mathrm{E}$ line is the golden section of the $\mathrm{D}$ line. The $\mathrm{D}$ line is the distance from the top of the head to the chin, and also e line is the width of the abdomen.

Scientists say that these lengths are for the ideal human body, where the suggested proportions will look natural and attractive to the human eye. Therefore a person with the golden proportions is a visually attractive person.

Some other proportions in the human hands that share the golden ratio include:
- Length of finger : Length of the first two sections of the finger

- Length of middle finger : Length of little finger

4.1.2 Human Face : An attractive human face, like the human body, seems to follow the golden ratio. The company Marquardt Beauty Analysis Inc, California, researches into human visual aesthetics to understand visual attractiveness. Fig.7. shows the perfect face as a mathematical computer model built up from a database of attractive faces. Both repose and smiling expressions can be seen in front and side views.

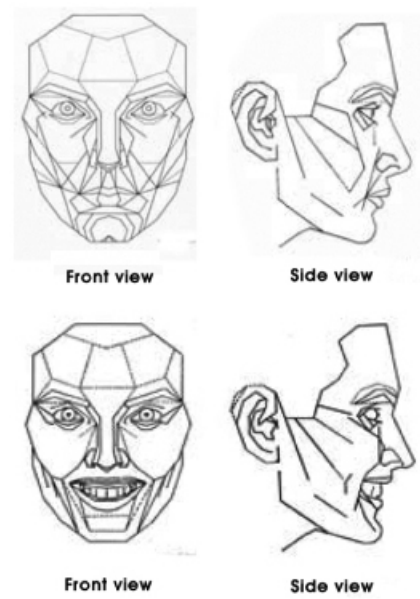

Fig.7. Mathematical models of perfect face

Some proportions in the face that share the golden ratio include:

- Length of face : width of face,

- Length of mouth : width of nose,

- Length of face : Chin to the eyebrows,

- Lips to the eyebrows : length of nose,

- Width of nose : distance between nostrils,

- Distance between pupils: distance between eyebrows.

Fig.8. shows an interesting comparison range between attractive and unattractive faces which shows how different faces match up to the perfect computer modeled face. Clients can download the perfect face mask and overlay it over their self photographs to compare the differences. The mask is useful as an aid when putting on make up, or for cosmetic surgery purposes.

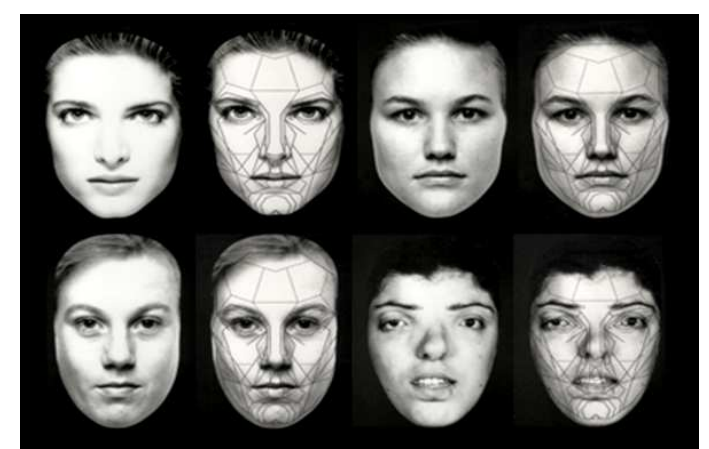

Fig.8. Visually attractive face range to unattractive face 
4.1.3 Human Teeth : The golden ratio can also be applied to the design of human teeth, and it is useful to know this subject when practicing dentistry. Dr. Levin of "Dr. Levin's Phi Dental Grid" lectures about his software and the applications of the golden ratio in dental aesthetics [14]. Fig.9. shows an image of a set of teeth that follows the ideal golden proportions. The golden proportions apply to the widths of the teeth as seen from the front and not the actual widths if they were individually measured.

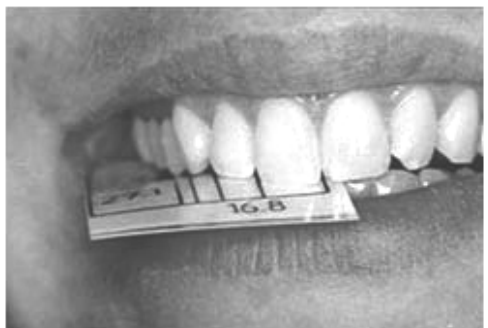

Fig.9. Golden Sections in human teeth

Fig.10. shows a client that had unattractive teeth due to the lack of gums or gingiva above the two front teeth. Using the golden ratio the dentist can use artificial gingiva to create a golden rectangle for the two front teeth. The before and after can be seen in the images. Dr. Levin is also the creator of the Golden Mean Gauge which will be reviewed in section 4.2.5.
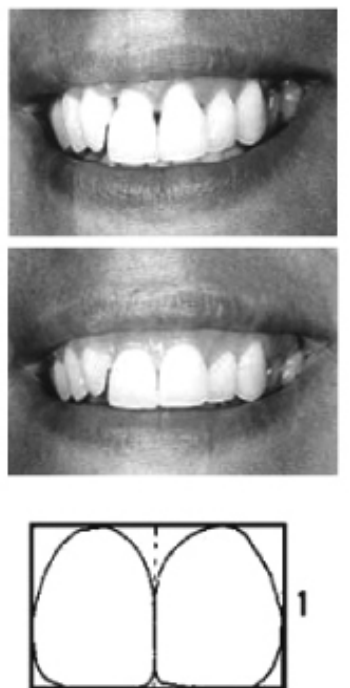

1.618

Fig.10. Gums fixed following golden rectangle

4.1.4 Plants : The golden ratio and the Fibonacci numbers were first seen in plants and nature. It was when this discovery was made that scientists and designers started to believe in this phenomenon. In botany or plant biology, the term "phyllotaxis" is the arrangement of the leaves/seeds of a plant [15]. The basic patterns of phyllotaxis are Distichous, Whorled, Spiral, and Multijugate. For plants that have spiral or multijugate phyllotaxis, the leaves/seeds grow one by one from the centre, each at a constant divergence angle close to the golden angle of $137.5^{\circ}$. Plants intuitively grow like this because the arrangement allows the plant to have optimal performance.
When leaves are arranged in rotation of golden angles, the plant can take in the maximum amount of sunlight [20].

Fig.11. shows an image of the Echinacea Purpura or the coneflower, a beautiful flower with its petals arranged in spirals. When the spirals are counted you can find that there are 55 spirals going clockwise and 34 spirals going anti clockwise. These two numbers are important numbers as they are consecutive Fibonacci numbers [3]. As shown in Section 2, these two Fibonacci numbers $34: 55$ give a ratio equal to the golden ratio.

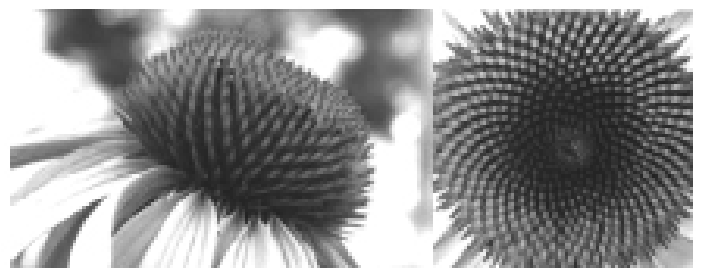

Fig.11. Spiral phyllotaxis in coneflower

All plants that have spiralling leaves/seeds seem to be based on the golden ratio, for example an ordinary pinecone will have 8 spirals clockwise with 13 spirals anticlockwise and as before 8:13 gives a result close to the golden ratio. Another example is a pineapple which has many spirals due to the hexagonal scales on the surface, with 5, 8, 13 and 21 spirals all increasing in steepness. Perhaps the most commonly chosen plant used to describe the beauty of the golden ratio in nature is the sunflower. Sunflower heads can be large, but however large the spirals within the seeds are always adjacent Fibonacci numbers. Fig.12. shows an image of a sunflower with the spirals illustrated.

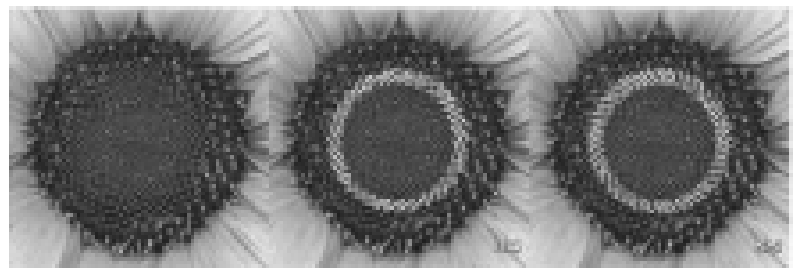

Fig.12. Spiral phyllotaxis showin in sunflower head

4.1.5 Shells: The Nautilus Seashell is the most popular shell and example when showing how the golden ratio relates to nature. The spiral seen from the shell is a logarithmic spiral, which is created by golden rectangles.

Fig.13. shows an image of the Nautilus and its shell as a cross section.
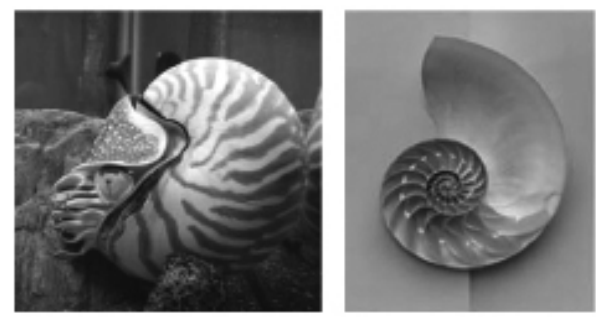

Fig.13. Logarithmic spiral in nautilus shell

The internal spirals grow with the animal and the internal 
chambers provide buoyancy in the water. The golden section spiral can be constructed using golden rectangles arranged in a spiral fashion.

By using the length of the sides of the rectangle as a radius for the arc of the spiral, the golden section spiral can be created. Other shells that have golden proportions include the Atlantic Sundial Shell, Moon Snail Shell and the Tibia Shell.

4.1.6 DNA : It is interesting to know that the golden ratio can also be found in the structure of DNA. DNA in cells is arranged as a double-helix, with spirals winding around each other. In between the DNA strands there are two grooves, the Minor groove and the Major groove, (See Fig.14.) It has been measured that the Major groove is 21 Angstroms wide and the Minor groove is 13 Angstroms wide. These two numbers are adjacent Fibonacci numbers which relate to the golden ratio.

If we look at the cross section of a DNA double-helix (Fig.10.), a regular decagon shape can be seen. The decagon is a special shape as the ratios between certain lines within the shape are equal to the golden ratio.

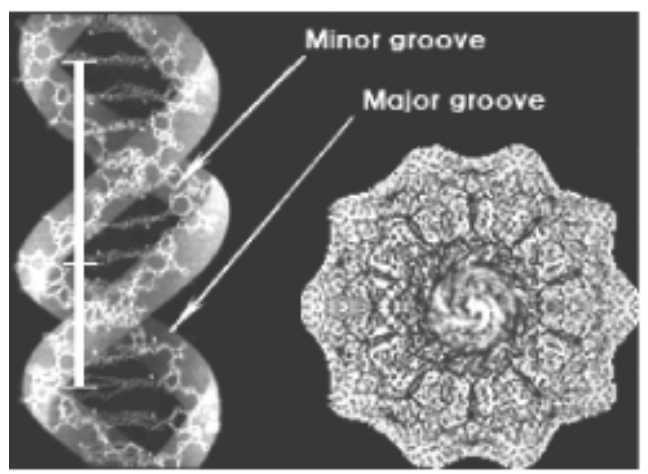

Fig. 14 Fibonacci numbers in DNA grooves

\subsection{In the Arts}

Designers, engineers, musicians, artists and poets have been using the golden ratio for a long time as a tool for creating beauty in their work. In this section there will be examples of pieces that have relationships with the golden ratio.

4.2.1 Paintings and Sculptures Perhaps the most famous piece of art that an artist has knowingly used the golden ratio in the painting is Salvador Dali's "The Sacrement of the Last Supper", (Fig.15.)

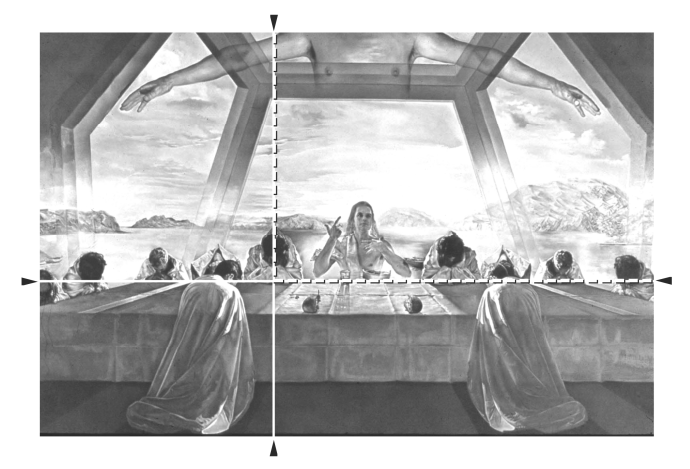

Fig.15. Golden Sections in The Sacrement of the Last Supper Dali has created the painting in a golden rectangle, with the table at a golden section from the height of the painting, and positioned the two disciples at a golden section from the two sides. The whole piece looks balanced as it is symmetrical and has used good proportioning techniques.

Dali has also painted windows in the background with the faces being from a dodecahedron solid. A dodecahedron is a platonic solid made up from 12 regular pentagons, which have golden ratio relationships in their proportions.

Another painting that exhibits a lot of golden sections is Piet Mondrian's "Komposition mit großer roter Fläche", (See Fig.16.). This painting has golden sectioning mixed with squares and rectangles of various sizes. The final piece looks attractive and somewhat mathematical as it is a piece that seems to have a lot of numbers.

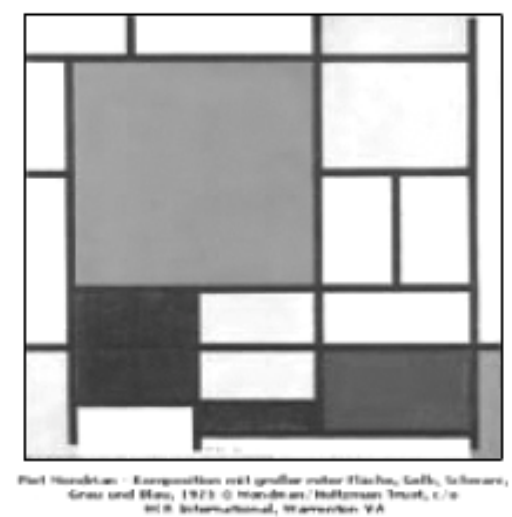

Fig.16. Golden rectangles in Mondrian's piece

Fig.17. shows an image of a sculpture called "Golden Ratio" contributed by an Australian sculptor Andrew Rogers. It is a fifty ton stone and gold installation situated in Jerusalem, which is a tribute to the Fibonacci numbers and the golden ratio. It can be seen from the left looking to the right there are blocks in units $1,1,2,3,5,8,5,3,2,1,1$, which is the Fibonacci sequence in palindrome fashion [17].

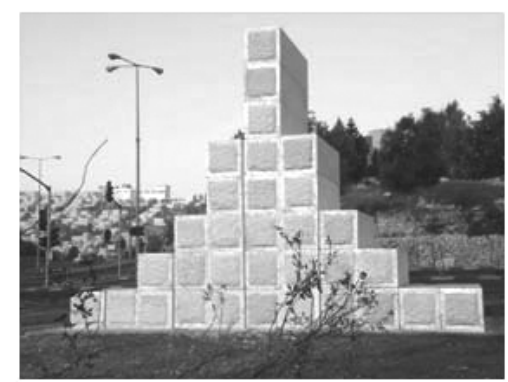

Fig.17. Golden Ratio Sculpture in Jerusalem

4.2.2 Architecture : The Parthenon is a beautiful piece of architecture built by the Greek sculptor Phidias for the Greek goddess Athena in 447-432BC. Many believe that Phidias used the golden ratio to proportion a lot of the dimensions of the temple; however more recently studies have been made that dispute the claim. The argument is that there is no evidence that Phidias employed the golden ratio into his architecture, even though the building seems to show it. Did Phidias naturally 
build the temple to the golden ratio proportions? Is golden proportioning a natural instinct? Fig.18. shows an image of the Parthenon with some illustrative lines showing the golden sections.

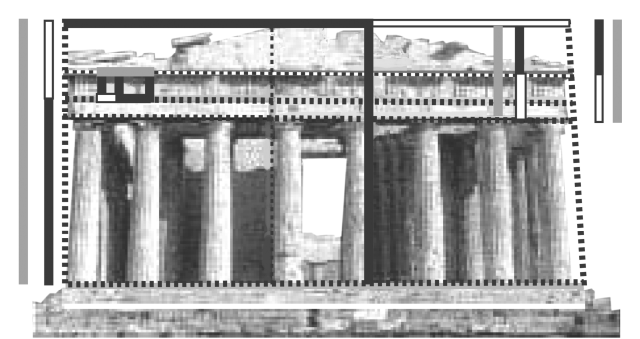

Fig.18. Golden sections in the Parthenon

The pyramids of Egypt are said to have been created with golden proportions. When a right angled triangle is formed with phi being the hypotenuse, the angle created is $51.83^{\circ}$. These matches with the angles of the slopes of the pyramids (see Fig.19.).
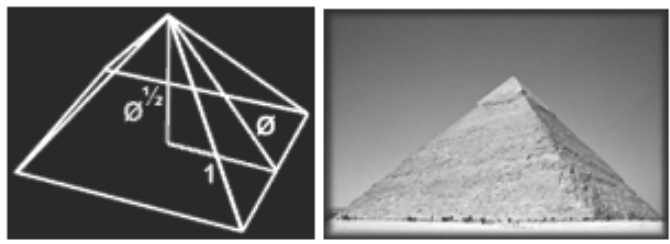

Fig.19. Golden Proportions in the Egypt Pyramids

Frederik Macody Lund, a historian who studied the geometry of gothic buildings, claimed that the Notre-Dame of Laon cathedral (1157-1205) was designed to the golden ratio, see Fig.20. He measured dimensions from the building and found many accounts of golden section proportioning. However, other scholars argue that architects knew nothing about the golden ratio as Pacioli's book "Da Divine Proportione" wasn't published until 1509. Is this another case of natural golden proportioning?

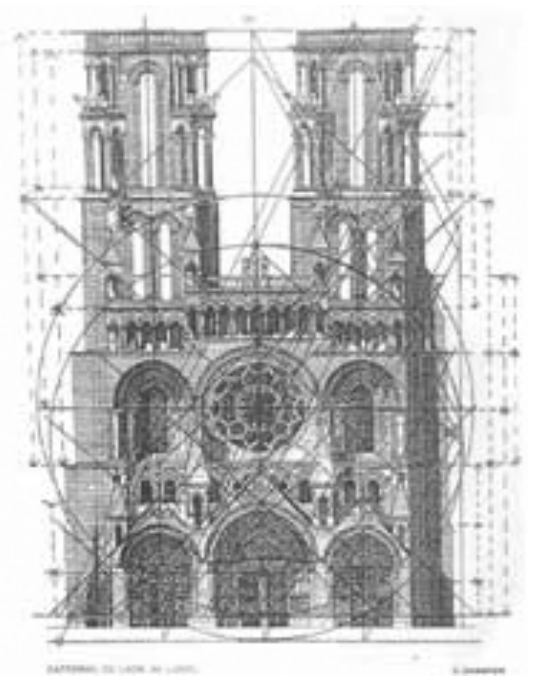

Fig.20. Golden Sections in Notre Dame of Laon
4.2.3 Music: Some musicians have gone as far as employing the golden ratio and Fibonacci numbers into their compositions. Musicians such as James Tenney with his piece "For Ann (Rising)" have used the golden ratio in places like between starting note intervals. Brian Transeau is another musician who has created a track " 1.618 " in homage to the golden ratio in his album "This Binary Universe". The golden ratio can be often found in the timing of musical compositions, such as the climax of songs will usually be near $61.8 \%$ in the song. In a 32 bar song this would mean the climax will be in the 20th bar.

It sits there, the raven, black as night, looking at me with its dark eyes in the dark night. Inspiration comes. Words form in my head. Evermore

4.2.4 Poetry : Poets have even used the golden ratio and Fibonacci numbers as a way to compose their poems. "Inspiration Comes (Fibonacci)" by Jim T. Henriksen, 2006, is a poem that uses the Fibonacci sequence 1,1,2,3,5,8,13,21,34 as a sequence for the amount of syllables used per line. The poem can be read below.

I
am
sitting
quietly,
listening for the
quiet noises in the darkness,
ages flying between the tall pine trees,

illusion created by the mind, made by shadows, the brain playing tricks on itself It sits there, the raven, black as night, looking at me with its dark eyes in the dark night. Inspiration comes. Words form in my head. Evermore

4.2.5 Products The golden mean gauge is a product designed by Dr. Levin which can always open up with its teeth to the golden section, (see Fig.21.). This gauge is useful for designers that want to create or measure golden sections visually. Dr. Levin has used his gauge in his dentistry job to create visually attractive teeth. The gauge is made of stainless steel and comes in various sizes.

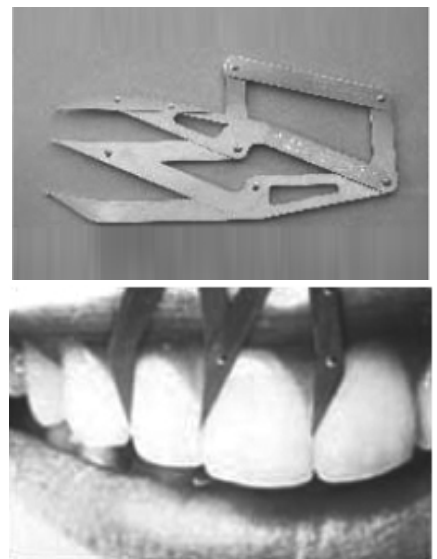

Fig.21. The golden mean gauge

Dr. Levin has also created another product, the Phi Matrix, (see Fig.22.). This is a piece of software designed for the dentistry market, as a computer alternative to the golden mean gauge. 
Users can upload photographs and place them under matrix lines that are positioned in golden section within each other. This software is useful to produce visually attractive teeth, and the calculations can all be done using the software.

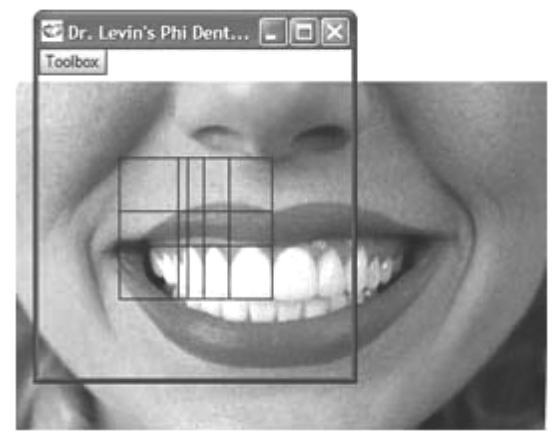

Fig.22. The Phi Matrix

Another product that uses the phenomenon of the golden ratio as a selling point is the Phi Chi Sticks. It is a curved stick that Master Rob Moses uses in his Tai Chi lessons to enhance the workout and keeps the body aligned and balanced. It is said to be "an easy and enjoyable way to move with grace and style both mentally and physically," (Moses,2009). (Fig.23.)

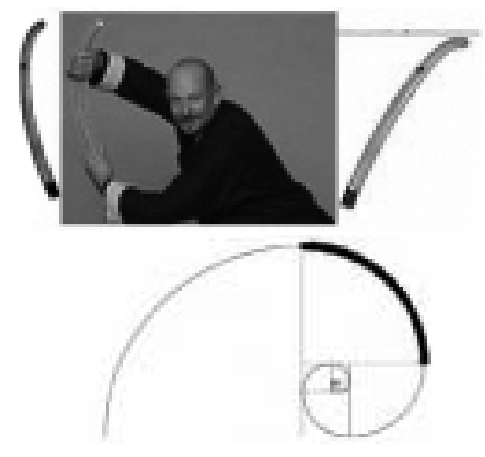

Fig.23. Phi Chi Sticks

There are not many products in the market that use the golden ratio as a selling point; however, there are many products that have used it so that they have been designed to be aesthetically better. One example is the Volkswagen Beetle.

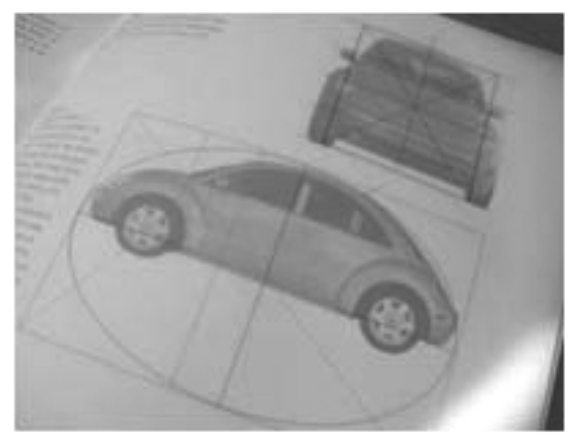

Fig.24. Golden Sections in VW Beetle

Many of the elliptical shapes of the body, windows, wheel arches, and the division lines for the doors and handles follow the golden ratio, (see Fig.24.). The credit card, books and envelopes are a few more examples where they are golden rectangles.

\section{METHODOLOGY}

In this work there will be an experiment to test if the application of the golden ratio in products affects product appeal. The experiment shall test the recipient's rate of appeal to certain proportions of designs. It shall test for consistency, as well as cognitive thinking. This experiment will involve appropriate research methods possibly in a form of a questionnaire. The questionnaire will be designed against the following specification:

- The questionnaire should not allude, imply or mention anything to do with the golden ratio. The recipient shall answer the questions without knowledge of the subject matter. This is so that there will be no biased answers and as every answer will affect the outcome of the results.

- The first question can be a variation of Fechner's experiment, a set of 10 different sized rectangles. The recipient shall answer the question by choosing the most visually attractive rectangle. The results will be tallied up and compared with Fechner's.

- The following questions shall be designed with images of consumer products with a series of varying proportions. The recipient shall answer the questions by choosing the image with the product that is most visually attractive. There shall be several of these questions, so several designs of common products shall be designed. The minimum is 3 designs.

- The images shall be large enough so that they are easily seen and compared.

- The designs shall be all the same shape, size and colour. The only difference is the proportioning of split lines, contours and edges.

- Different proportioning ratios shall be used including the golden ratio in the designs.

- The proportions in the designs shall differ enough so that they seem significant from each other.

- The golden ratio shall not be the proportion which is in the middle of the variations. The golden ratio shall be varied in the positioning of the proportion range i.e. sometimes the golden ratio can be the smallest proportion.

- There shall be a question that allows the recipient to draw their own proportions onto a design. This may be a division of a rectangle, or the placement of a subject above a background. There shall be at least 1 question that test the recipient's individual cognitive thinking.

- The questionnaire shall be asked to at least 50 recipients of any age and gender. The results should be the same for every recipient if the universal law of the golden ratio does exist.

- The results shall be expressed as tables and graphs, and reviewed against the key questions.

An example of the Fechner's rectangles can be seen below in 
Fig 25.

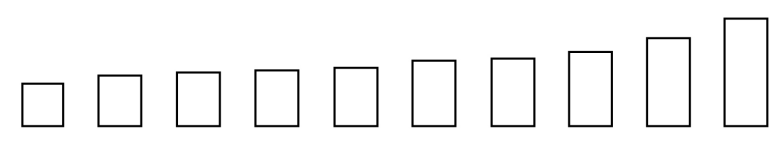

Fig 25. Fechner's rectangles from ratio 1:1 to 2:5.

\subsection{Questionnaire}

The following is the master copy of the questionnaire. The copy that was actually used in the investigation did not have the answers shown below. The answer with the dot rectangle is the answer with the golden proportions. The answer with the star is the image with the original proportions.

1. Which rectangle do you prefer?
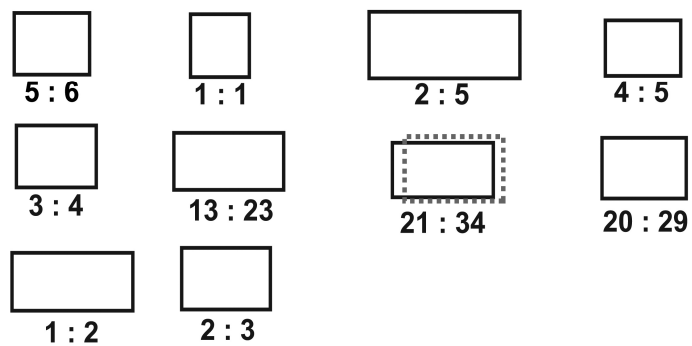

2. Which iron looks the most comfortable in proportion?
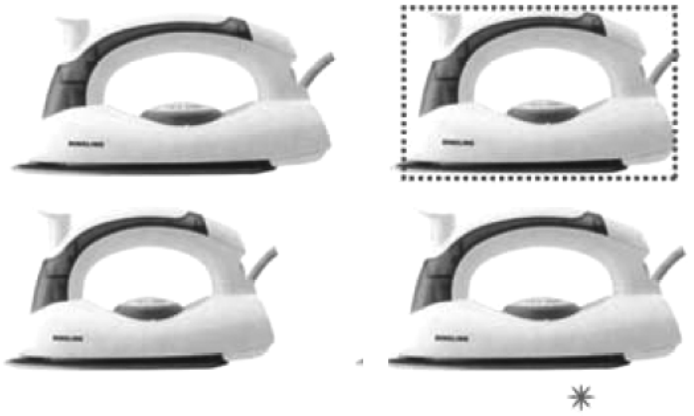

3. Which mobile looks the most comfortable in proportion?
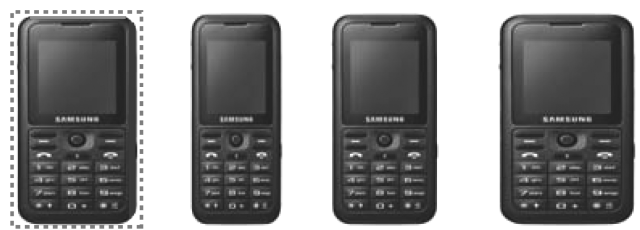

*

4. Which camera looks the most comfortable in proportion?
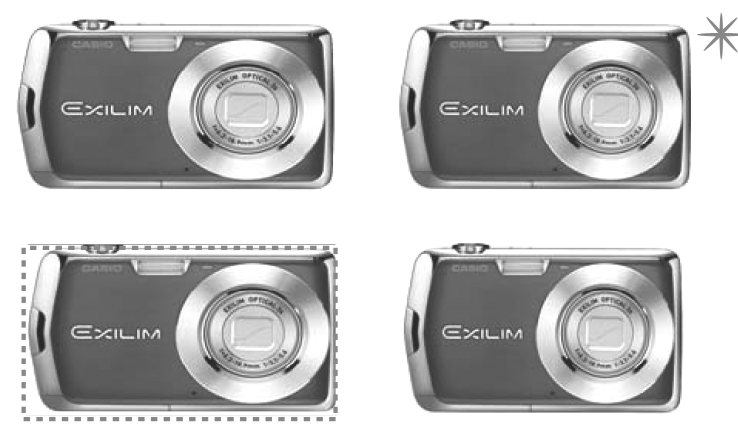

5. Which car looks the most comfortable in proportion?

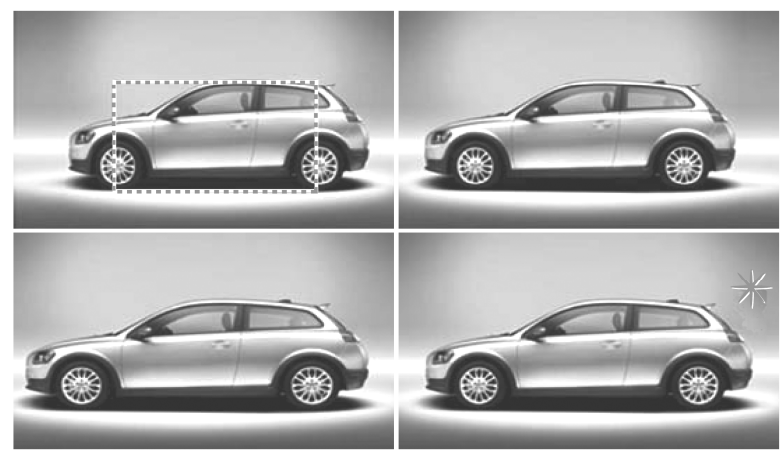

6. Which ipod looks the most comfortable in proportion?
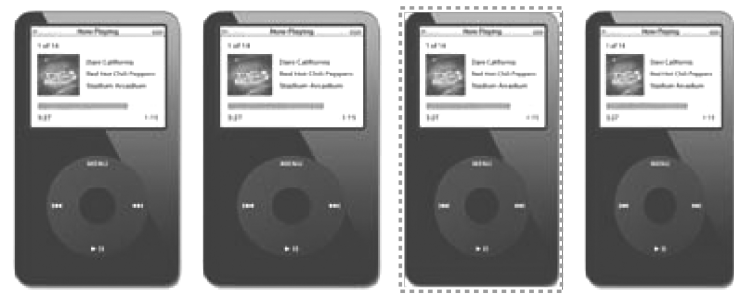

*

7. Place the hot air balloon in the sky where it feels right.

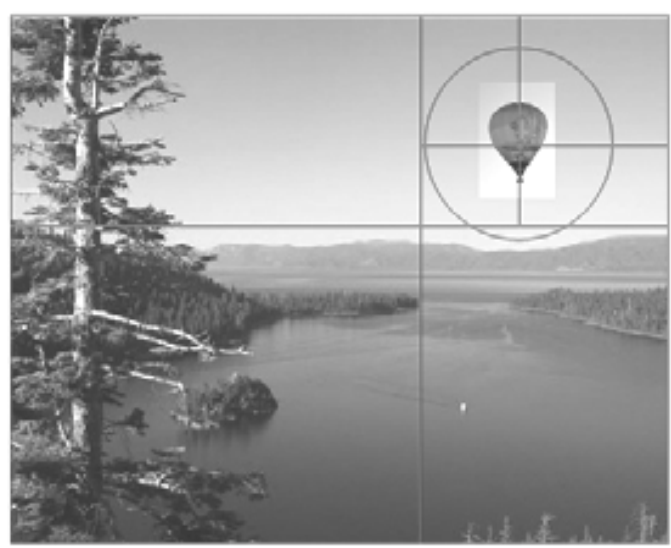

The hot air balloon has been positioned in the most comfortable position to the eye according to the golden section of proportioning. The hot air balloon is a separate cut-out for which the recipient of the questionnaire must place on the landscape according to where it "feels right". This question will test their cognitive intuition, and so it will really test if the answers are consistent among many recipients.

For questions $1-6$, the sequence order of small, original, golden, and big proportions were not consistent i.e. they were placed in a random order.

For the images in questions $2-6$, only one variable dimension was changed so that the recipient would see the subtlety of difference clearly. For these images only the $\mathrm{x}$ dimension was varied i.e. the height of the images were all kept the same.

For question 7, the quality of the answer will be judged by how 
close they are within the target circle. What $\%$ of total votes will be needed to be able to claim that the golden ratio is instinctive?

The answer may vary from question to question, but the prediction is that if the total votes is more than $50 \%$ on the golden rectangle, then the golden ratio can be deemed instinctive.

\section{SURVEY RESEARCH}

A total of 50 recipients were asked to complete the questionnaire, 25 male and 25 female. The questionnaire had a total of 7 questions. The answers that were given were given as a total free choice, and there was no biased answers given in any way. The following section is the collected results:

\subsection{Data Collection}

Question 1: Which rectangle do you prefer?

$\begin{array}{lccc} & \text { Male } & \text { Female } & \text { Total } \\ \text { Rectangle 1:1: } & 1 & 2 & 3 \\ \text { Rectangle 5:6: } & & 3 & 3 \\ \text { Rectangle 4:5: } & & 2 & 2 \\ \text { Rectangle 3:4: } & 1 & 5 & 6 \\ \text { Rectangle 20:29: } & 1 & 5 & 6 \\ \text { Rectangle 2:3: } & 5 & 5 & 10 \\ \text { Rectangle 21:34: } & 7 & 3 & \mathbf{1 0} \\ \text { Rectangle 13:23: } & 7 & & 7 \\ \text { Rectangle 1:2 } & 2 & & 2 \\ \text { Rectangle 2:5: } & 1 & & 1\end{array}$

Question 2: Which iron looks the most comfortable in proportion?

$\begin{array}{lccc} & \text { Male } & \text { Female } & \text { Total } \\ \text { Iron 1* : } & & & \\ \text { Iron 2: } & \mathbf{1 3} & \mathbf{1 0} & \mathbf{2 3} \\ \text { Iron 3: } & 9 & 12 & 21 \\ \text { Iron 4: } & 3 & 3 & 6\end{array}$

Question 3: Which mobile looks the most comfortable in proportion?

$\begin{array}{lccc} & \text { Male } & \text { Female } & \text { Total } \\ \text { Mobile 1: } & \mathbf{1 0} & \boldsymbol{8} & \mathbf{1 8} \\ \text { Mobile 2* : } & 5 & 7 & 12 \\ \text { Mobile 3: } & 10 & 10 & 20\end{array}$

Mobile 4:

Question 4: Which camera looks the most comfortable in proportion?

Male Female Total

Camera 1:

Camera 2*:

$\begin{array}{lll}17 & 15 & 32 \\ 8 & 10 & \mathbf{1 8}\end{array}$

Camera 3:

Camera 4:
Question 5: Which car looks the most comfortable in proportion?

$\begin{array}{lccc} & \text { Male } & \text { Female } & \text { Total } \\ \text { Car 1: } & \mathbf{3} & \mathbf{9} & \mathbf{1 2} \\ \text { Car 2: } & 10 & 14 & 24 \\ \text { Car 3: } & & & \\ \text { Car 4*: } & 12 & 2 & 14\end{array}$

Question 6: Which ipod looks the most comfortable in proportion?

$\begin{array}{lccc} & \text { Male } & \text { Female } & \text { Total } \\ \text { Ipod 1*: } & 5 & 6 & 11 \\ \text { Ipod 2: } & & & \\ \text { Ipod 3: } & \mathbf{1 4} & \mathbf{1 5} & \mathbf{2 9} \\ \text { Ipod 4: } & 6 & 4 & 10\end{array}$

Question 7: Place the hot air balloon in the sky where it feels right.

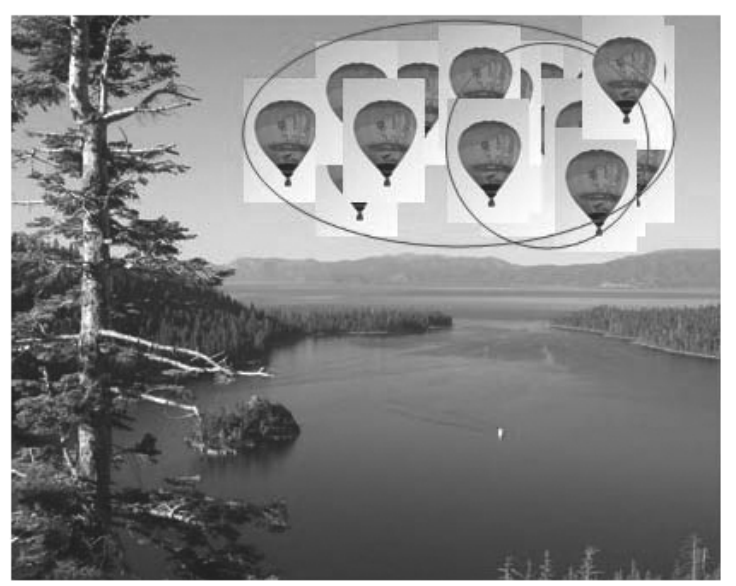

40 in the target circle

10 outside the target circle

\subsection{Summary}

- Question 1: Which rectangle do you prefer? It is interesting to see that the golden rectangle had got picked the most, with the slightly smaller rectangle having the same amount of votes too at 10 votes each. The rest of the votes were quite random when counted cumulatively, but it is clear that the males voted more to the larger rectangles and the females voted more to the smaller rectangles. Is there a reason for this pattern between the sexes? A total of $20 \%$ picked the golden rectangle in this question, which $70 \%$ were male.

- Question 2: Which iron looks the most comfortable in proportion? The golden rectangle had 23 votes which was the favourite. Iron 3 which is quite close to the golden rectangle had 21 votes. It is interesting here how there are no votes on the original iron. Is this a symbol for bad design? A total of $46 \%$ picked the golden rectangle, which $57 \%$ were male. The votes were quite even between the sexes.

- Question 3: Which mobile looks the most comfortable in proportion? The golden rectangle was picked 18 times, and the original mobile was picked 12 times. The most votes go to mobile 3 which looks like the middle proportion with 20 votes. 
No votes go to the largest sized mobile. A total of $36 \%$ picked the golden rectangle, where $56 \%$ were male. Does the golden rectangle look too big for a mobile phone in a generation where slimmer and smaller is better?

- Question 4: Which camera looks the most comfortable in proportion? Only two options were voted on in this question. The golden rectangle was picked 18 times and the original camera was picked 32 times. Does the golden rectangle look too big or do they prefer the more squared shape? Maybe they could pick the most natural looking camera as the circles may have turned ellipses in some of the answers. A total of $36 \%$ picked the golden rectangle, where $56 \%$ were female. For the first time the females have more votes on the golden rectangle, however, it is pretty much even.

- Question 5: Which car looks the most comfortable in proportion? The golden rectangle got 12 votes in this question, and car 2 the slightly larger sizes got 24 votes. The original car got 12 votes as well, and car 3 had no votes. It is not a surprise that car 3 had no votes because it does look rather big and out of proportion. The most votes go to car 2 which is the middle sized car. Here we see the trend where the males have voted on the larger longer sized cars, whereas the females have picked the smaller sized cars. Are the recipients choosing cars according to what they would drive in reality? A total of $24 \%$ picked the golden rectangle, where $75 \%$ were female.

- Question 6: Which ipod looks the most comfortable in proportion? Ipod 3 received 29 of the votes which presented an interesting split amongst responses.

- Question 7: Place the hot air balloon in the sky where it feels right. It is tough to see where all the votes were placed on the photo, but it was counted that 40 placed the hot air balloon somewhere within the target circle, closest to the golden position. 10 placed the hot air balloons close but in random positions. There were no individual male or female votes on this question. A total of $80 \%$ placed the hot air balloon within the target circle. Is an instinctive feeling to humans that we can visually place an object on a background because of what "feels right"?

There were collectively a possible 350 votes on all 7 questions. A total of 150 votes were voted correctly on the golden rectangle. That is a total of $43 \%$ of total votes. This is less than half of the total votes and it begs the question, is the golden ratio actually instinctive within nature?

\section{CONCLUSION}

The investigation was conducted successfully and the results were collected and analysed. The total votes collected that were correctly on the golden rectangle were $43 \%$. This is below our prediction so is it possible to say that the golden ratio has no real effect? If we look at which questions had the most golden rectangle votes on, we will find that it was 4 out of 7 questions. 1 out of the remaining 3 questions was a close match, and the remaining 2 had chosen other answers as the mode.

Question 7 was the question with the most golden rectangle votes on, and it was the only question where the recipient had to instinctively find the golden position. This result was quite surprising and does suggest evidence that people can "feel" what's right. However, when the same recipients were subject to a choice, not all of them can instinctively choose the correct answer. It is a possible that some of the questions had answers that were too similar to differentiate, but careful planning of the questionnaire had eradicated this problem. It is also possible that because of the skewing of some of the photos, it would look obvious that the skewed photo did not have good proportions, even though it was an attempt to skew it to the golden rectangle. The camera question was a good example of this, as the there were too many regular circles in the shape which meant that any skewing turned them into ellipses. Therefore for that point, the answers for that question can be ruled out. Even so, 132 golden rectangle votes out of 300 total votes equals to $44 \%$ which is still under the prediction.

It is therefore determined that the golden ratio used in proportioning isn't always the preferred choice. However, it is clear that the rectangles that are close to the golden rectangle will suffice. This result can be seen from question 1, where the 3 main rectangles 2:3, 21:34 and 13:23. The investigation shows that using the golden ratio to proportion designs will be useful, but there can be an allowance of $+/$ - a few units. The golden ratio can affect product appeal, as seen in some of the results; a badly proportioned product may not get any sales.

This investigation agrees with the following quote by Kimberly Elam, 2001 [5], "The purpose of Geometry of Design is not to quantify aesthetics through geometry but rather to reveal visual relationships that have foundations in the essential qualities of life such as proportion and growth patterns as well as mathematics. Its purpose is to lend insight into the design process and give visual coherence to design through visual structure. It is through this insight that the artist or designer may find worth and value for themselves and their own work." [5]. What Elam suggests in her book is that using a system such as golden proportioning, or any other valid geometric proportioning system, it will add some well-thought structure to the design, rather than a random choice of dimensioning. When limitations allow, the golden ratio can be useful.

\section{REFERENCES}

[1] Agarwal, R, 2001, "Golden Ratio in science, as random sequence source, its computation and beyond", Computers and Mathematics with Applications 56, 2008, pp.469-498.

[2] Devlin, K, The Math Instinct: Why You're A Mathematical Genius (Along With Lobsters, Birds, Cats, And Dogs), Thunder's Mouth Press, New York, 2005.

[3] Dunlap, R, The golden ratio and Fibonacci numbers, Singapore, London : World Pacific, 1997.

[4] Elam, K, Geometry of Design, Princeton Architectural Press, New York, 2001.

[5] GoldenNumber, 2009, "GoldenNumber.net", Available at: http://goldennumber.net [Accessed Jan 2009]

[6] GoldenMeanGauge, 2009, “TheGoldenProportion”, Availableat:http://www.goldenmeangauge.co.uk/index2.h tm [Accessed Jan 2009] 
[7] Knott, R, 2008, "The Fibonacci Numbers and Golden Section in Nature", Available at: http://www.mcs. Surrey.ac.uk/Personal/R.Knott/Fibonacci/fibnat.html [Accessed Apr 2009]

[8] Livio, M, 2002, "The golden ratio and aesthetics", Available at: http://plus.maths.org/issue22/features/ Golden [Accessed Jan 2009]

[9] Livio, M, The golden ratio: the story of phi, the extraordinary number of nature, art and beauty, Review, London, 2002.

[10] Mullard, P, 1999, "Proportions and use of the Golden Section in furniture design", Available at: http:// members.fortunecity.com/petemullard/mobaing.html [Accessed Jan 2009]

[11] Marquardt Beauty Analysis, 2009, "MBA California", Available at: http://www.beautyanalysis.com/index2_ mba.htm [Accessed Apr 2009]

[12] Padovan, Richard, Proportion: Science, Philosophy, Architecture. London: Taylor \& Francis., 1999. pp. 305306. ISBN 0-419-22780-6.

[13] Phi Chi Sticks, 2009, "Phi Chi Sticks", Available at: http://www.phichisticks.com/ [Accessed Apr 2009]

[14] PhiDental, 2005, "Dr. Levin's Phi Dental Grid by PhiMatrix”, Available at: http://www.phidental.com/ [Accessed Apr 2009]

[15] Phyllotaxis, 2009, "Phyllotaxis", Available at http://maven.smith.edu/ phyllo/ [Accessed Apr 2009]

[16] Thomas, B, Geometry in Pictorial Composition, Newcastle Upon Tyne, Oriel Press Limited, 1969.

[17] Vajda, S, Fibonacci and Lucas numbers, and the golden section: theory and applications, Chichester: Ellis Horwood, 1989.

[18] Wikipedia, 2009, "Golden Ratio", Available at: http://en.wikipedia.org/wiki/Golden_ratio [Accessed Jan 2009]

[19] Wikipedia, 2009, "List of works designed with the goldenratio", Available at: http://en.wikipedia.org/ wiki/List of works designed with the golden ratio [Accessed Jan 2009]

[20] Wikipedia (2009), "Phyllotaxis", Available at: http://en.wikipedia.org/wiki/Phyllotaxis [Accessed Apr 2009]

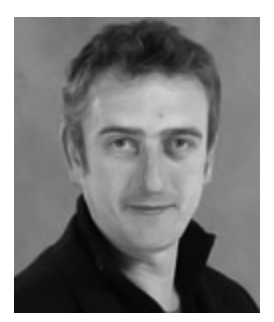

\section{Tom Page}

He obtained his BSc. and M.Phil in Engineering Design from Napier University, in 1988 and 1992. He obtained a $\mathrm{PhD}$ from the University of Hertfordshire in 2002. He is an external examiner on Engineering and Manufacturing programmes at Sheffield Halllam University. $\mathrm{He}$ is a visiting scholar at Iceland University and the University of Lapland in Finland and has been an external examiner on undergraduate fields in Product Design and Manufacturing Engineering at the University of East London. (2008 to present) $\mathrm{He}$ is a lecturer in Electronic Product Design in Department Design and Technology at
Loughborough University, 2003 to present. Among his main research areas are in the research and development of computer applications for design and technology education, logistics and supply chain management and electronic product design.

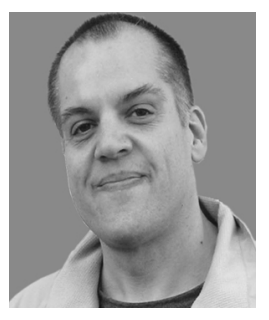

\section{Gisli Thorsteinsson}

He obtained his B.Ed. in 1980, in Design and Craft Education from the University of Iceland and his $\mathrm{PhD}$. from Loughborough University, Leicestershire, England in 2010. Gisli has been the Chairman of the Association of Icelandic Industrial Arts Teachers since 1995.

From 2000 he has been on the Board of 'Nordfo', the Pan Scandinavian co-operative researching Art and Design projects in Scandinavia. Gisli has been an Assistant Professor at the University of Iceland, since 1996, in the Department of Design and Craft Education. His main research focus has been on exploring the values of using Virtual Reality Learning Environment for supporting the development of ideation skills in students, in Icelandic schools.

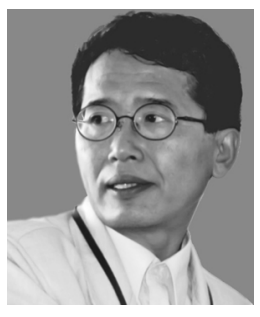

\section{Joong Gyu, Ha}

He obtained his B.A. and M.A. in Industrial Design from Hong-Ik University, Seoul, Korea, in 1985, 1987 respectively. He was associated with BioDesign Innovation Centre in Korea as CEO from 2003 to 2005 and was a visiting scholar in Sustainable Design in the Department of Design and Technology at Loughborough University, Leicestershire, England. He has won the 'Best Design Award' from the 2009 Exhibition of Invited International Designers, 16 countries around the world's representative industrial and environmental designer participating in Busan, Korea. He is a professor of Industrial Design in the Department of Art Education at Gyeongsang National University, Korea, from 1988 to present. His research interests include the sustainable product design education for industrial designers and environmental designers. 